\title{
The Impact of High Intensity Interval Training and Anthropometric Changes in Women
}

\author{
Joan A Cebrick Grossman* \\ Department of Kinesiology, The University of Scranton, USA
}

\author{
*Corresponding author: : Joan A Cebrick Grossman, Department of Kinesiology, \\ The University of Scranton, 237 Jefferson Ave. Scranton, PA 18510, USA, Tel: 570- \\ 941-4721; 570-941-6209 Email:joan.grossman@scranton.edu
}

Received Date: April 01, 2019

Published Date: April 18, 2019

\section{Mini Review}

This mini review highlights three recent pilot studies that have shown promising results in women, specific to the impact of high intensity interval training (HIIT) and changes in anthropometric measurements in women. The first study observed the effects of HIIT on anthropometric, body composition and resting metabolic rate (RMR) measurement changes in sedentary, overweight, middle-aged women over a 12-week period [1]. The subjects ( $\mathrm{N}=9$ ) were female volunteers $(51.3 \pm 5.5 y e a r s ; 77.20 \pm 6.12 \mathrm{~kg}$ body wt; $\mathrm{BMI}=28.9 \pm 2.1 \mathrm{~kg} / \mathrm{m}^{2}$; mean+SD) who exercised five out of seven days for 12 weeks. The exercise program (15.0 $\pm 3.0 \mathrm{~min})$ consisted of five different exercise routines that included total body, lower extremity, yoga, cardio and abdominal segments. Relative body fat was measured via air displacement plethysmography, along with five anthropometric measurements and $\mathrm{RMR}\left(\mathrm{V}_{\max }\right.$ metabolic system) prior to and after 12 weeks. Dependent t-tests probed for significant differences $(\mathrm{p} \leq 0.05)$. Pre-post body weight and RMR were not statistically significantly different. However, four of the five pre-post anthropometric measurements were significantly reduced for the abdomen, hips, waist and thigh measurements $(97.79 \pm 5.11$ vs. $91.95 \pm 5.64 ; 104.90 \pm 5.33$ vs.100.84 \pm 5.33 ; $84.25 \pm 7.57$ vs. $80.26 \pm 7.36 ; 62.99 \pm 5.08$ vs.59.18 $\pm 4.82 \mathrm{~cm}$, mean \pm SD). This work is suggestive that HIIT contributes to significant anthropometric reductions, in the abdomen, hips, waist and thighs, which may decrease chronic disease development, such as obesity, cardiovascular disease and diabetes.

The mode and duration of exercise necessary to change body composition and reduce weight remains debatable. A second study compared the effects of HIIT and traditional exercise, (walking) on anthropometric and body composition measurement changes in post-menopausal women over a 12-week period [2]. The study subjects $(\mathrm{N}=18)$ were sedentary, overweight, post-menopausal females, who were randomly assigned into one of two exercise groups. Both groups exercised five out of seven days for 12 weeks.
The resistance group $(\mathrm{n}=8)(54.3 \pm 7.3$ years; $\mathrm{BMI}=28.0 \pm 2.1 \mathrm{~kg} /$ $\mathrm{m}^{2}$; mean $\pm \mathrm{SD}$ ) exercised for $15.0 \pm 3.5 \mathrm{~min}$, which consisted of five different exercise routines including upper and lower extremity, a cardio segment, yoga and abdominal exercises. The walkers $(n=10)$ $\left(56.6 \pm 5.2\right.$ years; BMI $\left.=29.2 \pm 2.6 \mathrm{~kg} / \mathrm{m}^{2}\right)$ exercised for $40.0 \pm 5.0$ min at $65 \%$ of their age-predicted maximum heart rate. Relative (percent) body fat was measured via DEXA scan, along with five anthropometric measurements (waist, abdomen, hips, thigh and biceps), all of which were taken prior to and after 12 weeks. Independent sample t-tests were probed for differences, $(\mathrm{p} \leq 0.05)$. Although no statistically pre-post measures were determined, most likely due to the small sample size, all outcome variables indicated promising trends of significance. This work provides a foundation for future comparisons of HIIT and traditional exercise, regarding anthropometric and body composition measurement changes in sedentary, overweight, post-menopausal females.

The third study was a 16-week behavioral weight loss program that incorporated wearable activity sensors to facilitate self-monitoring exercise [3]. Participants $(\mathrm{N}=11)$ were obese, post-menopausal, sedentary women (59.5 \pm 3 .3years; $\mathrm{BMI}=32.08 \pm 2.18 \mathrm{~kg} / \mathrm{m}^{2} ;$ mean $\pm \mathrm{SD}$ ) who were randomly assigned to a HIIT or an endurance exercise group. Both groups followed a calorie-restricted diet, attended monthly in-person meetings, weekly weigh-ins and electronic check-ins to review behavioral skills, and monitored their exercise via a Fitbit Charge HR. Adherence to exercise programs, assessed with the Fitbit sensor, was used to determine feasibility. The results of the study indicated that participants in the HIIT group $(\mathrm{N}=6)$ adhered to their program, while 3 of 5 adhered to the Endurance program. Participants in the HIIT group lost twice as much weight as those in the Endurance group ( $8.7 \%$ vs. $4.3 \%$ of initial body weight), and lost an additional $15.24 \mathrm{~cm}$ of body mass. In addition, only the HIIT group significantly changed pre-post measures of fat mass $(41.6 \pm 3.46 \mathrm{~kg}$ vs. $29.12 \pm 5.59 \mathrm{~kg})$, fat-free mass $(42.99 \pm 3.31 \mathrm{~kg}$ vs. $42.58 \pm 4.6 \mathrm{~kg})$ 
and BMI ( $30.67 \pm 2.16$ vs. $27.76 \pm 2.76)(\mathrm{p} \leq 0.04)$, respectively. The findings support the feasibility and potential effectiveness of HIIT for weight loss and body composition changes in obese, postmenopausal women, and indicate that additional investigation of this approach is warranted to reduce post-menopausal chronic disease risk.

Although the sample sizes for each of these studies were small, the outcome measures provided promising results for sedentary, overweight and obese women later in life. A primary barrier to exercise for women is time, and a short duration exercise program, such as HIIT may be a possible answer to this a barrier. These works were supportive in identifying the impact of HIIT, resulting in favorable anthropometric, body mass and body composition changes reducing chronic disease risk, in this at-risk population.

\section{Conflict of Interest}

No conflict of interest.

\section{References}

1. Cebrick Grossman JA (2016) The impact of short-duration interval training on anthropometric, body composition and resting metabolic measurement changes in overweight women: a pilot study. Gazzetta Medica Italiana 175(1-2): 34-39.

2. Cebrick Grossman JA, Payne EK (2016) A randomized comparison study regarding the impact of short-duration, high-intensity exercise and traditional exercise on anthropometric and body composition changes in post-menopausal women: a pilot study. Post Reproductive Health 22(1): 14-19.

3. Grossman JA, Arigo D, Bachman JL (2018) Meaningful weight loss in obese postmenopausal women: A pilot study of high-intensity interval training and wearable technology. Menopause 25(4): 465-470.

\section{Acknowledgement}

None. 